\title{
Agrobiodiversité et pratiques agricoles dans le pays Jbala (Tafza et Bellota)
}

Interaction entre ethnobotanique et linguistique

Agrobiodiversity and farming practices in the Jbala region (Tafza and Bellota):

interaction between ethnobotany and linguistics

\section{Alexandrine Barontini et Younes Hmimsa}

\section{(2) OpenEdition}

Journals

Édition électronique

URL : https://journals.openedition.org/ethnoecologie/3217

DOI : $10.4000 /$ ethnoecologie.3217

ISSN : 2267-2419

Éditeur

Laboratoire Éco-anthropologie

Référence électronique

Alexandrine Barontini et Younes Hmimsa, « Agrobiodiversité et pratiques agricoles dans le pays Jbala (Tafza et Bellota) », Revue d'ethnoécologie [En ligne], Supplément 1 | 2017, mis en ligne le 17 octobre 2017, consulté le 19 novembre 2021. URL : http://journals.openedition.org/ethnoecologie/3217 ; DOI : https://doi.org/10.4000/ethnoecologie.3217

Ce document a été généré automatiquement le 19 novembre 2021.

Revue d'ethnoécologie est mis à disposition selon les termes de la licence Creative Commons Attribution - Pas d'Utilisation Commerciale - Pas de Modification 4.0 International. 


\title{
Agrobiodiversité et pratiques agricoles dans le pays Jbala (Tafza et Bellota)
}

\author{
Interaction entre ethnobotanique et linguistique
}

Agrobiodiversity and farming practices in the Jbala region (Tafza and Bellota): interaction between ethnobotany and linguistics

Alexandrine Barontini et Younes Hmimsa

\section{Objectif et aire d'étude}

1 Cet article vise à présenter des résultats d'une recherche interdisciplinaire menée dans deux sites différents, présentant des agroécosystèmes traditionnels de montagne et qui relèvent de la région $\mathrm{jbala}^{1}$. L'étude porte essentiellement sur le croisement de données ethnobiologiques et de données linguistiques dans le but de mettre en évidence les spécificités de ces deux sites. La recherche s'est effectuée dans le cadre d'un Projet International de Coopération Scientifique (PICS) franco-marocain et avec l'aide du Centre Jacques Berque (CJB) de Rabat.

2 Ainsi, au cours de la réalisation de l'étude nous avons focalisé notre intérêt sur la mise en évidence de l'état de l'agrobiodiversité de ces sites à travers un inventaire complet des cultures et des variétés locales. Dans ce cadre, plusieurs sorties de terrain ont été réalisées sur deux périodes différentes (octobre 2013 et avril 2014).

Lors de ces missions, nous avons eu l'occasion de rencontrer des agriculteurs et des habitants dans les deux sites. À chaque rencontre, un ensemble de question leur a été posé en ce qui concerne l'agrobiodiversité caractéristique du site sans oublier la part des pratiques et usages.

4 L'étude a été réalisée sur deux sites (douar ou dchar) différents en pays (Figure 1). 
Figure 1 : Localisation des sites d'étude au nord occidental du Maroc

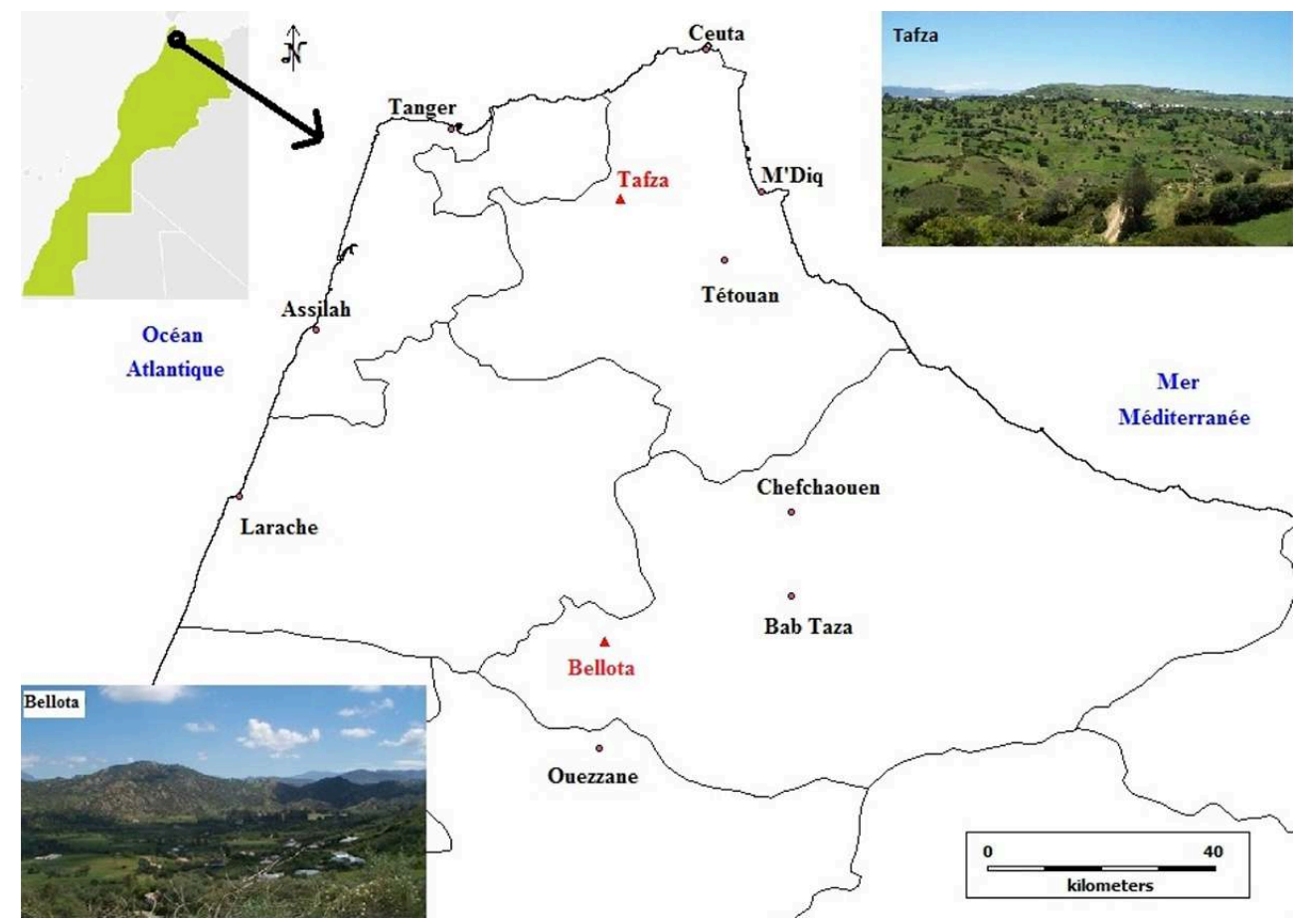

Carte réalisée avec le logiciel DIVA-GIS (version 7.5.0, free software disponible à http://www.divagis.org/

5 Le village de Tafza. Il fait partie de la commune rurale de l'Anjra (Province Fahs-Anjra). C'est un village qui se situe sur une route tertiaire à $5 \mathrm{~km}$ du souk hebdomadaire de Khmiss d'Anjra. Le village abrite une population jəbliyya (montagnarde) reconnue localement par le nom des Barqōqiyyen ${ }^{2}$, en faisant référence à l'abondante production des prunes dans ce village. Sur le sommet d'une colline, le village de Tafza s'étend sur une superficie de 350 ha et regroupe 70 ménages (Direction de la Statistique 2004).

Le village de Bellota. Il fait partie de la commune rurale de Brikcha (Province d'Ouezzane). Le village est situé sur la route nationale (RN13) reliant Chefchaouen à Ouezzane à $1 \mathrm{~km}$ du souk hebdomadaire de Sebt de Brikcha. Le territoire du douar est traversé par l'Oued Ouaghan et par deux grands fleuves Khandak Errha et Khandak Msiouine. Situé à l'intérieur d'un versant montagneux, il est très étendu, d'où l'aspect d'un habitat peu dense avec une architecture principalement traditionnelle constituée de 98 ménages et 594 habitants (Direction de la Statistique 2004).

7 Du point de vue de la structuration du territoire au niveau des deux sites, on peut faire la distinction entre deux zones : un noyau habité (situé en colline à Tafza et au centre du versant à Bellota) et des zones périphériques cultivées (deux versants à exposition nord et sud à Tafza et des parcelles éparpillées dans le versant à Bellota). Le noyau du village regroupe des habitations organisées en quartiers (deux quartiers à Tafza et quatre quartiers à Bellota) correspondant à des lignages familiaux différents (quatre noms de famille sont majoritaires sur le village) et relativement dispersées sur la superficie du village. Le paysage dans les deux sites est caractérisé par la présence de cultures et de vergers, on observe une grande diversité d'exploitations agricoles, y compris des champs de céréales ou de légumineuses associés à des vergers de fruitiers (figuier, olivier, vigne). 


\section{Méthodologie}

Dans le cadre de ce travail, des campagnes de prospections ont été menées dans le but de rencontrer des agriculteurs soit chez eux dans les douars, dans les champs, ou lors des marchés hebdomadaires. Nous avons utilisé une approche ethnobiologique pour la récolte des données selon une approche mixte croisant les observations participantes réalisées sur les sites avec les données issues des enquêtes directes et des discussions semi-directives menées avec les agriculteurs. La première finalité était de recueillir des informations concernant un inventaire exhaustif de l'agrobiodiversité qui existe dans les sites sélectionnés. En particulier, nous avons porté plus d'intérêt aux plantes cultivées, connues et identifiées par les agriculteurs au niveau infra-spécifique, afin de mettre en lumière les variétés locales (ou cultivars) utilisées qui ne sont pas sélectionnées ni diffusées par les pépiniéristes, le département de l'agriculture ou les marchands de semences (Doré \& Varoquaux 2006). La diversité perçue et utilisée localement peut être analysée et interprétée en fonction d'une approche ethnobotanique.

9 La seconde finalité était de recueillir par ces conversations, un échantillon linguistique des variétés de parlers arabes en usage sur les deux sites. Le corpus a aussi permis l'établissement d'une liste des différents types de fruits et arbres fruitiers nommés (culture, variétés locales ou cultivars).

10 Les enquêtes ont été menées, sur les deux sites, par Younes Hmimsa (Y.) et Azzeddine El Azouzi (A.), à l'époque étudiant en master (M2, à la Faculté des Sciences de l'Université Abdelmalek Essâadi de Tétouan) et lui-même originaire de la région. Les conversations portaient sur la diversité agraire, les fruitiers n'étaient donc pas la seule culture évoquée. On ne visera pas une comparaison exhaustive, tant dans les conditions d'interaction que dans les profils des interviewé-e-s.

11 Ces derniers étaient soit des connaissances ou des proches de l'un ou l'autre des intervieweurs, soit des personnes rencontrées dans les villages, les marchés ou les champs. Au total, nous avons eu l'occasion de discuter avec huit hommes (quatre à Tafza et quatre à Bellota) et quatre femmes (à Bellota). Alexandrine Barontini, a pour sa part assisté aux enquêtes, enregistré et transcrit les corpus afin d'en présenter les principales caractéristiques phonologiques ainsi que la richesse lexicale illustrant l'agrobiodiversité locale.

12 Cette enquête à caractère transdisciplinaire permet ainsi d'enrichir le champ de l'ethnobotanique tout en produisant in situ des données inédites sur le plan strictement de la linguistique.

\section{Site de Tafza (Caidat de L-Anjra) :}

$\mathbf{H 3}$ et $\mathbf{H 4}$ : hommes, 55 ans et 63 ans, tous les deux du village de Hlatet, rencontrés sur la route entre Touilech-Tafza-Hlatet.

Si A. : homme (70 ans), Tafza, interviewé seul et avec Si M.

Si M. : homme (84 ans), Tafza, interviewé avec Si A.

\section{Site de Bellota (Caidat de Brikcha) :}

F1 : femme, 50 ans environ, interviewée chez elle, après qu'elle nous ait accompagnés sur le marché.

Fa : femme âgée, marché. 
Ha et $\mathbf{H b}$ : hommes, 60 et 66 ans, marché.

H1 : homme, 75 ans, dans un champ.

H5 : homme, 65-70 ans, dans un champ.

\section{Principaux traits linguistiques marquants de nos enregistrements à Tafza et Bellota}

Les deux zones d'enquête correspondent à deux zones dialectales de la région des parlers jbala : L-Anjra étant proche de Tétouan (Vicente 2000) et Bellota étant proche d'Ouezzane (Vicente et al. 2017, Benitez Fernandez 2016). Les parlers jbala («montagnards ») font partie des parlers sédentaires dits pré-hilaliens, c'est-à-dire ceux de la première période de l'arabisation du nord de l'Afrique (fin $\mathrm{VII}^{\mathrm{e}}$-VIII ${ }^{\mathrm{e}}$ siècles). Ils font ainsi partie des quatre régions arabisées avant l'arrivée des Hilaliens, avec le Trara et le nord constantinois en Algérie et le Sahel tunisien (Marçais 1956). Les parlers de ces régions présentent des traits communs liés notamment à une forte influence du substrat berbère et à un contact qui perdure (au Maroc et en Algérie surtout). Les parlers jbala reflètent aussi les liens étroits entretenus à l'époque de l'Espagne musulmane entre la région et Al-Andalus. Toutes ces caractéristiques les distinguent des autres parlers arabes du Maroc. Les parlers jbala restent toutefois partiellement étudiés et décrits (Vicente 2000, Moscoso 2003, Guerrero Parrado 2014) au regard de leur diversité.

Les traits linguistiques présentés ici sont loin d'être exhaustifs, mais ils nous indiquent des différences et des convergences entre les deux zones de recherche. Ils montrent aussi le maintien chez les personnes âgées que nous avons interviewées de traits caractéristiques des parlers jbala. La description se focalise principalement sur la variation phonétique et indique quelques aspects morpho-syntaxiques particuliers, en suivant les critères d'analyse dialectologique détaillés par D. Caubet (2000-2001). Au niveau lexical, les données indiquent la richesse terminologique concernant les principaux arbres fruitiers. Ce choix trouve sa justification dans le fait que c'est au niveau des arbres fruitiers que l'on a recensé le plus de diversité au niveau des deux sites d'étude (Figure 2). En outre, les arbres fruitiers constituent l'une des composantes des agroécosystèmes traditionnels du $\mathrm{Rif}^{3}$ en général et de ces deux sites en particulier. Il s'agit d'éléments importants et caractéristiques de ces agroécosystèmes traditionnels. Ainsi, dans la partie ci-dessous, en s'intéressant à la composante fruitière nous analysons d'une manière détaillée trois types de fruitiers (figuier, prunier et poirier) qui sont marquées par leur importance en termes de présence, de diversité variétale voire même d'intérêt économique ${ }^{4}$. En effet, les deux sites connaissent pendant la saison de production de ces trois fruitiers un grand mouvement de commerçants qui viennent en voiture pour chercher les fruits à vendre sur les centres urbains (Tétouan, Tanger, Ouezzane, Chefchaouen ...).

Figure 2 : Comparaison entre le nombre de culture et de variétés par culture au niveau des sites d'étude et à l'échelle du Rif

\begin{tabular}{|c|c|c|c|c|c|c|c|}
\hline \multirow{2}{*}{ Site } & \multicolumn{4}{|c|}{ Nombre de types de culture } & \multicolumn{3}{c|}{ Nombre de variétés } \\
\cline { 2 - 7 } & Céréale & Légumineuse & Fruitier & Céréale & Légumineuse & Fruitier \\
\hline Tafza & 4 & 7 & 13 & 5 & 9 & 61 \\
\hline Bellota & 4 & 8 & 11 & 5 & 10 & 43 \\
\hline Rif & 6 & 8 & 15 & 10 & 10 & 218 \\
\hline
\end{tabular}




\section{Phonétique : réalisation des consonnes et voyelles}

\section{Réalisation du /q/}

La réalisation est sourde, comme dans les autres parlers pré-hilaliens, et l'on retrouve les deux variantes recensées au Maroc : [q] et [ $\left.{ }^{[}\right]$.

\section{Le /q/ est réalisé [q] à Tafza :}

l-barqūq (la prune), Eonq ḥmām (cou de pigeon), qədd əṛ-rāa (comme la tête).

À Bellota, il est réalisé la plupart du temps [ $\left.{ }^{2}\right]$ chez les hommes âgés comme chez les femmes, et [q] chez quelques hommes rencontrés au marché. La réalisation glottale [ $\left.{ }^{?}\right]$ attestée dans les parlers citadins de Fez ou Taza et dans certains (anciens) parlers juifs (Sefrou, Chefchaouen), ou encore en usage seulement chez les femmes (Tetouan), est considérée comme un trait en voie de disparition (Vicente 2000, Aguadé 2008), avec notamment la perte de prestige des parlers pré-hilaliens. Il semble ainsi que les jeunes générations (de par leur mobilité liée à la scolarité) aient tendance à éviter certains traits trop distinctifs, telle cette réalisation ${ }^{[}{ }^{3}$ (Benitez Fernandez 2016). Le corpus recueilli ici ne concerne, toutefois que des locuteurs plutôt âgés. Parmi les occurrences relevées :

${ }^{3}$ Omḥ (blé), l-wa $a^{3}$ (le temps), əl-bar ${ }^{3} \bar{u}^{2}$ (la prune), ${ }^{2}$ ədd hāyda (comme ça / de cette taille),

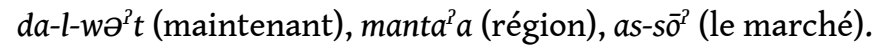

H1 : u l-bar $\bar{u}^{2}$ ha lo-khal ha lə lo-byađ̣ ha lə lo-ḥməṛ. əl-anwāe bar ${ }^{2} \bar{u}^{2}$. (...) hìya hādi ssmīya. șāfi (...) žābu hādə. mnayn žābu hād-əl-filāḥa. m əl-mo ${ }^{2}$ rīsā̄. ${ }^{2} \partial d d$ hāyda. wa ìla Ţāmər mlīh. wa lāçin ma la-yțỉb ši bəçri.

« Et la prune voilà la noire, voilà la la blanche, voilà la la rouge. Les variétés des prunes (...) C'est ça le nom. C'est tout (...) ils ont amené ce. D'où ils ont amené cette culture. De Mokrisset. Comme ça. Et s'il donne des fruits c'est bien. Mais il ne mûrit pas tôt. »

\section{Réalisation du /k/}

Le / $\mathrm{k} /$ peut être réalisé [k] ou [ç]. La réalisation [ç] est beaucoup plus présente à Tafza. Tafza :

H4 : ka-ytēeb bəçri hādəç / kūl lli ḃ̀iti kūl dìk-əl-bārāka dyal-um « il mûrit tôt celui-là / mange ce que tu veux mange de leur bénédiction » Si M. : wa lākin ya xā-y! / hādīç, diç, hādāç / ka-yeəllmu-ç Mais mon frère ! / celle-là, cette, celui-là / ils t'enseignent

Bellota :

F : kađāliç (aussi)

H1 : lāçin ma la-yțīb ši bəçri.

\section{Réalisation du /b/}

21 Outre la réalisation [b], on rencontre quelques occurrences d'une réalisation [ $\beta]$, à Bellota seulement.

Fa : $d \bar{a} \beta a$ (maintenant) / $d$ ət țțā $\beta$ (en terre)

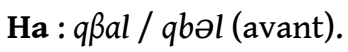




\section{Réalisation du /t/}

\section{Tafza :}

Si A. : kāyən əs-səbŢi ki yqūlu l-u / Ţbāṛk ḷ̂āh / šhar əs-səțŢa / ŢəlŢ myāŢ sna

Il y a le sebti (ceuti) comme on l'appelle / Dieu soit loué / le mois de juin / trois cents ans.

\section{Bellota :}

F1 : Ţ-Ţāmanīnāt / ēh dāba əz-zayŢūn kŢər

Les années 1980 / oui maintenant il y a plus d'oliviers

H1 : ha n-nūe Tु-Ţāmər

Voilà la variété qui donne des fruits

Tandis que la réalisation [于] apparaît plus rarement et seulement dans les enregistrements à Bellota :

F1 : ma kfā̄-hum š / dāba mā̄ālān əl-yūma / əz-zī̄ EāwŢāni

Elle ne leur suffit pas / maintenant par exemple aujourd'hui / l'huile aussi.

\section{Réalisation du /d/}

Réalisé $[\mathrm{d}]$, le /d/ est aussi très souvent réalisé [đ], à toutes les positions. Il s'agit làaussi d'une fricatisation secondaire due au substrat berbère ${ }^{6}$.

\section{Tafza :}

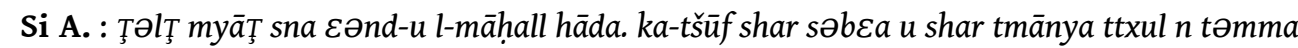

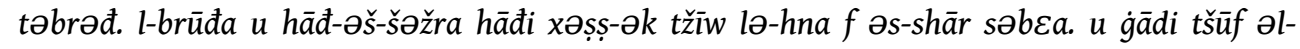

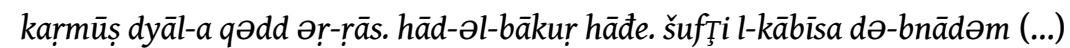

«Il a trois cents ans cet endroit. Tu vois au mois de juillet et d'août tu entres là-bas tu sens le frais. La fraîcheur et cet arbre-ci il faut que tu viennes ici au mois de juillet. Et tu verras ses figues (grosses) comme la tête. Ce figuier-ci. Tu as vu la tête des gens (...)»

H3 : u عənd-na z-zaytūn d əd-ğdūđ

Et nous avons l'olivier des ancêtres

Si A. : blīnsi. blīnsi đa hāđa hūwa lli:: (...)

La prune blinsi. La prune blinsi c'est celle qui (...)

\section{Bellota :}

F1 : hāō e:: s-sīnīn hāđi / amma dāba l-wa $a^{2} T$ hāđa

Ces années-là / mais maintenant en ce moment

H5 : əs-səbca u r ud

La figue «sept et repose-toi »

H5 : wa lākin tbārk əḷ̂̄h e:: l-bākōra dyāl-a ${ }^{2} \partial d d$ hāyđa

Mais louange à Dieu sa figue-fleur est (grosse) comme ça.

Revue d'ethnoécologie, Supplément 1 | 2017 


\section{Réalisation du /ḍ/}

\section{Tafza :}

Y : u l-lūbya l-bayḍa ūla l-həmra (et les haricots blancs ou rouges)

H3 : l-bayța (les blancs)

Si A. : la l-blinsi k-yzi byət

Non la prune blinsi devient blanche

\section{Bellota :}

Fa : hād-əd-ğwāyah lli țāwìyin n təht

Ces coins qui sont éclairés en bas.

on l'a aussi rencontrée à Ourtzagh (Ziamari \& Barontini 2008) :

Tafza :

H3 : dīk-lə-g̀lēđ̣ (la grosse-là)

Si M. : u l-hafri u l-bìyuạ u l-qūṭi (et la figue hafri et la blanche et la qoti)

Bellota :

F1 : $\bar{a}$-yžəmEu l-anwāe d zaytūn lə-byađ̆

Ils rassemblent les variétés d'olives vertes (claires)

H1 : l-bayđa i / l-bar ${ }^{2} \bar{u}^{2}$ ha lə-khal ha lə lə-byađ̆

La figue blanche / et la prune voilà la noire voilà la blanche.

\section{Réalisation du /ž/}

Il est réalisé [ž], mais aussi [̌̌]. Comme cela a été souligné par Vicente (2000 et 2007) et Aguadé (2003), cette réalisation apparaît en position intervocalique lorsque le phonème est géminé, en position initiale et aussi au contact de certaines consonnes comme $\mathrm{n}$ et r. Concernant son comportement avec l'article, nous trouvons [əd-ğ], un phénomène décrit par Vicente pour Anjra (2000) : «L'assimilation de l'article définit /l-/ avant /ž/ fait que l'apparition de cette gémination et sa dissimilation consécutive sont très fréquentes, exemples : $d-\bar{g} l a ̄ l ə b$ « les jellabas » $(. ..) »^{7}$

$[\check{z} \check{z}]=>[\check{g}]$

H3 : əz-zaytūn d əd-ğđūâd (l'olivier des ancêtres)

Si M. : lingāṣ $d$ əd-ğiyyāf. iyyeh (...) əd-ğ $\bar{u} \varepsilon$

La poire « de l'étrangleur ». oui (...) la faim

Si A. : ğənžlān (sésame)

Bellota :

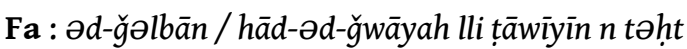

Les petits pois / ces coins qui sont éclairés en bas

Ha : əd-ğarda

Le jardin

$\mathrm{Hb}:$ : $l-\varepsilon \bar{a} m d$ əd-ğū $\varepsilon$

L'année de la faim.

$31 \quad[\check{z}]=>[g]$ 
Bellota :

Fa : gāzət mudda

Il s'en est passé du temps.

\section{Amuiissement du /h/}

On constate l'amuïssement de $/ \mathrm{h} /$ seulement dans les pronoms, phénomène que l'on retrouve à Anjra (Vicente 2000) et plus généralement dans les parlers pré-hilaliens.

34 Tafza :

H4 : $\varepsilon$ əənd-əm (chez eux)

Si A. : dīk-əl-bārāka dyal-um / əl-karmū̄ṣ dyāl-a

Leur bénédiction / ses figues

Bellota :

F1 : əšš̌ižər dyāl-əm / hūwa l-mədxōl d əs-sāna kull-a

Leurs arbres / c'est le revenu de l'année entière

H5 : wa lākin tbārk əlḷāh e:: l-bākōra dyāl-a ${ }^{2} \partial d d$ hāyđa

Mais louange à Dieu sa figue-fleur est (grosse) comme ça.

\section{Sifflantes et chuintantes}

On relève une altération instable des chuintantes en sifflantes:/ž/ réalisé [z] et /š/ réalisé [s], à Tafza chez Si A. et Si M.

Si A. : hăza qdìma (une chose ancienne)

Si A. : dìk-əz-zī̄ kull-si hūwa s-sūmūm ā šššrîf

Cette huile c'est que du poison mon cher

Si A. : si-nās buržwāzīyiñ (des gens bourgeois)

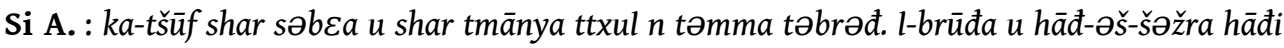

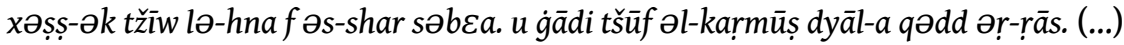

$\mathrm{Tu}$ vois au mois de juillet et d'août tu entres là-bas tu sens le frais. La fraîcheur et cet arbre-ci il faut que tu viennes ici au mois de juillet. Et tu verras ses figues (grosses) comme la tête.

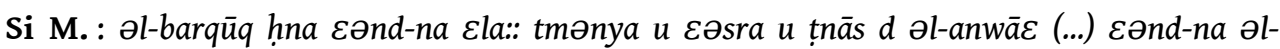
uwwliya al-blinsi

Les prunes nous on en a huit ou dix ou douze variétés (...) on a la première la blinsi

Si A. : hūwa lli ka-yțēb l-uwwlāni

C'est celle qui mûrit en premier

Si A. : la l-blinsi k-yzi byəț.

Non la prune blinsi devient blanche.

\section{Richesse des réalisations vocaliques}

On note une certaine richesse vocalique dans les réalisations des voyelles brèves et longues.

Tafza :

țobli, tabli, šižər, ənwāe/unwā $\varepsilon / a n w \bar{a} \varepsilon$

žūž bì-him

Eənd-əm 
dyāl-um

Bellota :

əl-farq bīnāt-him

šəžar, bukri, nōe/nū $\varepsilon$

əร̌-šižər dyāl-əm.

mə $n-n \bar{\varepsilon} \varepsilon$ əlli $\varepsilon \partial n d-\partial m . .$.

Avec parfois l'apparition d'une imala (altération de [a] en /e/ ou /i/) finale.

Tafza :

Angeles Vicente l'avait déjà relevé pour Anjra, et notait qu'elle n'y apparaît qu'en fin de mot et avant une pause (Vicente 2000). C'est le cas pour cette occurrence :

Si A. : hād-əl-bākur hā đe

Cette figue-fleur-ci.

Bellota :

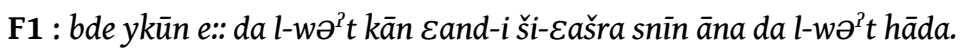

[...] b ən-nəsba l-manța $\bar{a}^{2} a$ dyāl-na hnāye

Ça a commencé à ce moment-là j'avais quelque chose comme dix ans en ce temps-là.

[...] par rapport à notre région ici.

\section{Morphologie et syntaxe}

\section{Le préverbe de la conjugaison préfixale}

Le préverbe de la conjugaison préfixale est $\mathrm{k}(\mathrm{a})-$, présent partout. Mais à Bellota on a aussi des occurrences de $\bar{a}$ - et la-, formes caractéristiques des parlers jbala.

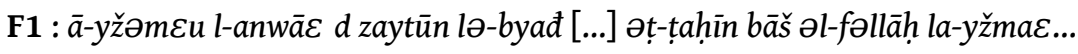

Ils rassemblent les variétés d'olives vertes [...] la mouture pour que le paysan rassemble...

H1 : wa lāçin ma la-yțỉb ši bəçri.

Mais il ne mûrit pas tôt

Fa : u la-tžì-na ġālya ḥnāya

Et ça nous revient cher à nous.

\section{Prépositions}

On note l'usage de la préposition directionnelle $n$.

Tafza :

Si A. : ttxul n təmma təbrəđ

Tu entres là-bas tu sens le frais.

Bellota :

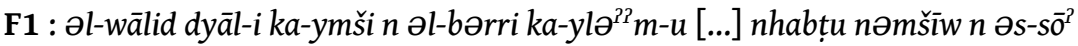

Mon père va dans l'olivier sauvage il le greffe [...] nous descendons nous allons au marché

Fa : hād-əd-ğwāyah lli țāwīyīn n təht

Ces coins qui sont éclairés en bas. 


\section{Les variétés d'arbres fruitiers et de fruits nommés}

40 Dans cette partie de notre étude, nous mettons en évidence les données traitant de la diversité des fruitiers (cf. Figure 3 et extraits du corpus). Ainsi, vu l'importance de celle-ci et le très grand nombre d'arbres fruitiers à inventorier à travers les agroécosystèmes des deux sites, nous nous limitons à l'analyse de la diversité de trois fruitiers, auprès des agriculteurs, en révélant les différentes variétés locales en se basant sur des noms vernaculaires.

41 Au niveau sémantique et lexical on notera la diversité terminologique utilisée pour nommer les arbres et les fruits en particulier pour le figuier, le prunier et dans une moindre mesure le poirier dans les deux sites. Sur le plan étymologique, les termes relevés ont été comparés aux sources des deux principaux dictionnaires d'arabe marocain issus du fond Colin : Iraqui Sinaceur 1993 et Prémare 1993-1999.

La taxinomie vernaculaire des variétés relève de divers procédés et champs sémantiques. Par comparaison et métonymie d'aspect, de couleur et/ou de propriétés (positives ou négatives), en référence à des toponymes ou ethnonymes, à des usages particuliers, des objets domestiques, des animaux, d'autres fruits ou végétaux.

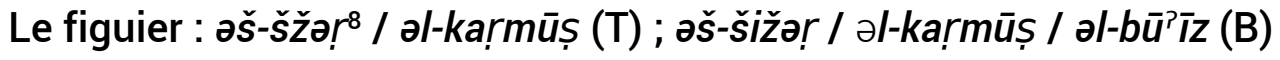

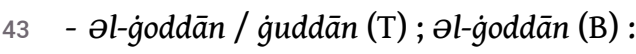

Colin (Iraqui Sinaceur 1993) donne pour traduction «Figue noire» et renvoie à « šąri. »

- əl-gūzi (T) :

Colin (Iraqui Sinaceur 1993) donne pour traduction «qui a la forme d'une noix » et précise que le terme s'applique à une espèce de figue ou de citron.

- əl-ḥofri / hafri (T) :

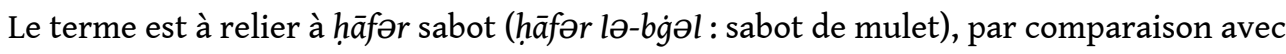
la taille et/ou la forme du sabot.

- al-bākor / bākur (T) :

Colin (Iraqui Sinaceur 1993) indique pour ce terme, «1. Figues fleur qui se gâtent vite et ne peuvent être séchées pour être conservées; figues précoces et de grande taille produites par certains figuiers donnant deux récoltes / toute chose sans valeur réelle, qui n'a qu'un temps ; méprisable ; bonne à rien. 2. Paroles injurieuses, malsonnantes. » Différents sens et usages qui ont en commun une dimension péjorative. Dans le contexte de notre enquête, il s'agit bien d'une variété nommée de figuier qui produit des figues fleurs.

- əl-ḥorši (T) : de ḥrəš, rugueux.

- Eonq ḥmām (T) ; Eon ${ }^{?}$ ḥmām (B) : cou ou gorge de pigeon, en rapport avec sa teinte.

- əs-səbŢi (T) : de Səbta (Ceuta).

- əz-zrəq (T) : le bleu, pour la population locale, c'est une couleur qui tient du bleu pour tendre vers le vert clair.

- əs-shïli (T) : diminutif de facile.

- əl-bìuự / lə-byəđ̆ (T) ; l-bayđị (B) : le blanc.

- əț-țobli (T) ; əț-țobli / əț-țabli (B) : de țbəl, tambour, percussion, en rapport avec sa forme.

- l-qūṭi (T) ; əl-º̄ṭi (B) : 
Prémare (1993) mentionne ce terme en référence aux parlers jbala, «l-qōtete / l-qōṭe d əlEarab variété de figue blanche ». Il est probablement lié à qōt / qawt: aliment, nourriture; aliment nourrissant; ou encore le blé dans la région Tanger-Anjra, toujours selon Prémare (1993).

- lə-h̆mər (T) ; əl-hamrri (B) : le rouge.

- əl-məssāri (B) :

Colin (Iraqui Sinaceur 1993) mentionne "l-məstāri : variété de figue", tandis que Prémare (1993) indique (à Tétouan) «l-məssāri variété de figue précoce [= l-məstāri] ». Ce dernier renvoie aussi à lə-mdar (région jbala) «variété de figue précoce blanche et noire » et à mEalləm mūs (région jbala) « variété de figue précoce ».

À rapprocher peut-être aussi du nom de la tribu bni məstāra, et du territoire qu'elle occupe (ou occupait) dont on suppose que la variété citée est originaire.

- əl-fərzāwi (A et B) :

Prémare (1993) indique "variété de figue (noire)». Le sémantisme de la racine a rapport avec le choix, la distinction, la préférence.

- əs-səmri (B) : le brun.

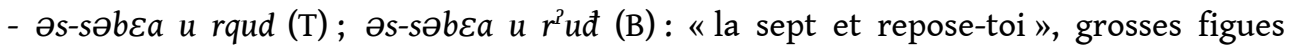
charnues (sept suffisent largement à être rassasié).

- əl-bərrāni (T) : de l'étranger.

Pour deux variétés citées de figues nous ne trouvons pas, à ce stade, de traduction ni d'explication :

- lə-ršān (T)

- əl-Eonbēz (T)

\section{Le prunier : əl-barqūq $(\mathrm{T})$; əl-bar' $\bar{u}^{\mathrm{P}}$ (B)}

Il est intéressant de noter que əl-bərqūq (lui-même calqué du grec praekokhion « fruit précoce ») a donné le mot abricot en français (en passant par le provençal, l'italien, le catalan, l'espagnol et le portugais). Aujourd'hui, əl-barqūq désigne la prune dans la plupart des parlers arabes nord-africains, à l'exception de quelques parlers algériens dans lesquels il désigne encore l'abricot (Marçais 1911, Pruvost 2017). Le glissement de sens n'est guère étonnant dans la mesure où ces deux fruitiers font partie de la famille des Rosaceae et le nom scientifique de l'abricot est Prunus armeniaca (prunier d'Arménie). L'abricot est donc généralement əl-məšmāš/əl-məšməš̀ , sauf pour la région jbala qui utilise le terme $\partial n-n \dddot{\imath}^{10}$.

- əl-blinsi (T) = əș-șfar / əș-șfifar (le jaune) :

Pour blinsi, Colin (Iraqui Sinaceur 1993) indique "variété de prune de petite taille, de couleur jaune très pâle ou blanche. litt. ${ }^{11}$ de Valence Bālinnsya ».

Pour cette même variété, l'appellation l-barqūq $d$ əd-dwāri est aussi apparue (T) : $d w \bar{a} r i$ pluriel de dūro, écu (emprunt à l'espagnol), en rapport avec la couleur de la prune.

«Si A. : hād-əl-barqūq əș-șfar. ās ka-yqūlu l-u hādāk?

Cette prune jaune. Comment on l'appelle celle-ci?

Si M. : e::h barqūq hna nqūlu l-u l-barqūq d əd-dwāri »

Ah oui une prune ici on l'appelle la prune des écus.

- lə-h̆mimar / əl-hūmorr / hūmar (T) lə-ḥmər (B) : le rouge.

- əl-khịhal / əl-kūhal / lə-kḩəl (T); lə-khal (B) : le noir.

- əl-bərtqiz / əl-portqiz / əl-portqēz (T) : 
Littéralement du Portugal, Prémare (1993) mentionne «l-bortqēze » comme une «variété de prune noire».

- aș-șayfi (T) :

De șêf/ṣayf, été. Colin (Iraqui Sinaceur 1993) donne pour traduction «1. bien exposé au soleil, ensoleillé. 2. d'été (fruits) », et Prémare (1993) indique « qui a rapport avec l'été ; estival (fruit)».

- $\operatorname{rr}$-rūmi (T) : l'européen.

- əd-dahbi / əd-dohbi (T) : qui a la couleur, l'éclat de l'or.

- lə-hrïbi (T) :

Il s'agit de la forme diminutive de l'adjectif harbi «qui est énergique, diligent et efficient (personne) », selon Colin (Iraqui Sinaceur 1993).

- əl-fārūq / fārūx / fārūç (T) :

Colin (Iraqui Sinaceur 1993) et Prémare (1993) mentionnent dìk fārūq: coq à double crête (porte-bonheur), appellation probablement en rapport avec la couleur et/ou l'aspect de cette prune. Une autre traduction, suggérée par F. Corriente, pourrait être «qui s'ouvre facilement », selon le sémantisme de la racine frq (qui a rapport avec le fait de séparer, diviser).

- barqūq ən-nsāra (T) :

Littéralement «la prune des chrétiens», les chrétiens ici étant les Romains de l'antiquité (comme pour karmūṣ ən-nsāra = l-həndīya : figue de barabarie).

- lə-byađ̣ (B) : le blanc.

\section{Le poirier : lingāṣ $(\mathrm{T})$; bū-عwiyyəd / lingāș (B)}

Selon Marçais (1911), l'origine du mot lingāṣ (de ižžāṣ) est araméenne et on le trouvait

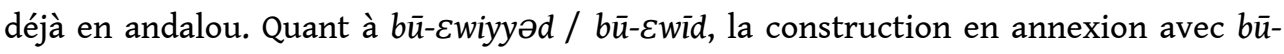
( " père de ») signifie « Ce/celui qui a, ayant » associé au diminutif du nom $\varepsilon \bar{u} d$ «bois » est à comprendre comme « ayant un bâtonnet comme queue » (Ibid.).

- ər-rūmi (T) : l'européen.

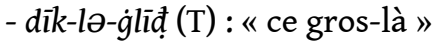

- əl-məski (T) :

Prémare (1993) indique pour le terme məski « qui a le parfum du musc, musqué », mais aussi «muscat (poires, raisins blancs - grain allongé et peau fine -, oranges)». L'adjectif réfère enfin à une teinte de jaune, « chamois clair » (Ibid.).

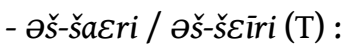

Littéralement chevelu/poilu, ou filandreux. Colin (Iraqui Sinaceur 1993) indique que le terme peut désigner une variété de figue «de taille moyenne allongée en poire de couleur noire violette; $c f$. goddāni » (voir plus haut), ou bien (à Tanger) une variété de poire.

- əl-xođ̈ri (T) : le vert.

- lingāṣ d əd-ğiyyāf $(\mathrm{T})$ : « la poire de l'étrangleur » cf. žîfa. Qui étouffe, étouffement

- lingās $d$-əl-xərrāy (șgīiwrīn! « elles sont très petites ») (T) : « la poire du chieur », poire très petite et de mauvaise qualité.

- lə-kbār $(\mathrm{T})$ : les grandes.

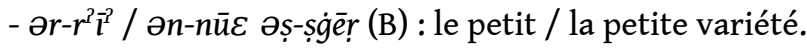

- ən-nū $\varepsilon$ Tु-Ţāmər (B) : la variété abondante / bonne (à point) à manger. 


\section{Discussion et conclusion}

Dans le cadre d'une approche interdisciplinaire, à travers ce travail nous avons mis en miroir les points de vue de l'ethnobiologie et de la linguistique, en partageant un même corpus pour aboutir à des constats complémentaires. Dans ce cadre, les résultats conçus par les travaux ethnobiologiques ont montré que l'agrobiodiversité, au niveau des deux sites d'étude, est très riche et diversifiée. Il est important de préciser que l'agrobiodiversité dans ces deux sites est représentée par la diversité des espèces végétales cultivées, essentiellement basées sur des variétés "traditionnelles" dites locales ou de terroir "bəldiyya ", constat qui est attendu puisqu'il a été déjà mis en évidence au niveau des agroécosystèmes du Rif (Hmimsa \& Ater, 2008). L'agrobiodiversité est constituée au niveau du site de Tafza par un total de 24 cultures pratiquées à base de 75 variétés nommées, alors qu'à Bellota, elle est représentée par 23 cultures pratiquées à base de 58 variétés nommées (Figure 2). Ce constat nous permet de confirmer que le site de Tafza est très important du point de vue de la diversité biologique agricole. À l'intérieur de cette agrobiodiversité, nous avons focalisé notre intérêt essentiellement sur la composante fruitière pour les raisons mentionnées plus haut. En effet, les arbres fruitiers, d'après les perceptions des agriculteurs, sont structurés en types nommés (figuier, prunier, poirier ...) à l'intérieur desquels on fait la distinction entre des variétés (Figures 3 et 4). La variété est l'unité sur laquelle s'exercent les pressions de sélection anthropiques. En ce sens, l'agriculteur détermine l'effectif de chaque variété dans la richesse fruitière.

Figure 3 : Richesse variétale des arbres fruitiers dans les sites d'étude par rapport au Rif

\begin{tabular}{|c|c|c|c|c|c|c|}
\hline \multirow{2}{*}{ Espèce } & \multirow{2}{*}{ Nom commun } & \multicolumn{2}{|c|}{ Nom vernaculaire } & \multicolumn{3}{|c|}{ Nombre de variétés } \\
\hline & & Tafza & Bellota & Tafza & Bellota & Rif \\
\hline Ficus carica & Figuier & \multicolumn{2}{|c|}{ šəžar, krəm, karmūș } & 17 & 11 & 133 \\
\hline Vitis vinifera & Vigne & dālya & ḩkəm & 11 & 4 & 21 \\
\hline Prunus domestica & Prunier & \multicolumn{2}{|c|}{ berqōq } & 8 & 2 & 13 \\
\hline Pyrus communis & Poirier & lingāș & lingāș, bū-દwiyyəd & 4 & 2 & 11 \\
\hline Malus pumila & Pommier & \multicolumn{2}{|c|}{ Teffăh } & 6 & 0 & 8 \\
\hline Punica granatum & Grenadier & \multicolumn{2}{|c|}{ roṃ̣mān } & 3 & 3 & 7 \\
\hline Olea europea & Olivier & \multicolumn{2}{|c|}{ zītūn } & 2 & 5 & 7 \\
\hline Prunus armeniaca & Abricotier & ən-nīš & əl-məšmāš & 1 & 1 & 5 \\
\hline Citrus sinensis & Oranger & \multicolumn{2}{|c|}{ bortūqāl, lə-čĩn } & 4 & 2 & 4 \\
\hline Opuntia ficus-indica & Figue de barbarie & \multicolumn{2}{|c|}{ həndiyya } & 2 & 1 & 2 \\
\hline Cydonia oblonga & Cognassier & \multicolumn{2}{|c|}{ sfəržəl } & 1 & 1 & 2 \\
\hline Prunus dulcis & Amandier & \multicolumn{2}{|c|}{ lawz } & 0 & 0 & 2 \\
\hline Juglans regia & Noyer & gawz & nwa & 1 & 1 & 1 \\
\hline Prunus avium & Cerisier & \multicolumn{2}{|c|}{ habb əl-mlūk } & 1 & 0 & 1 \\
\hline
\end{tabular}


Figure 4 : Liste récapitulative des variétés nommées

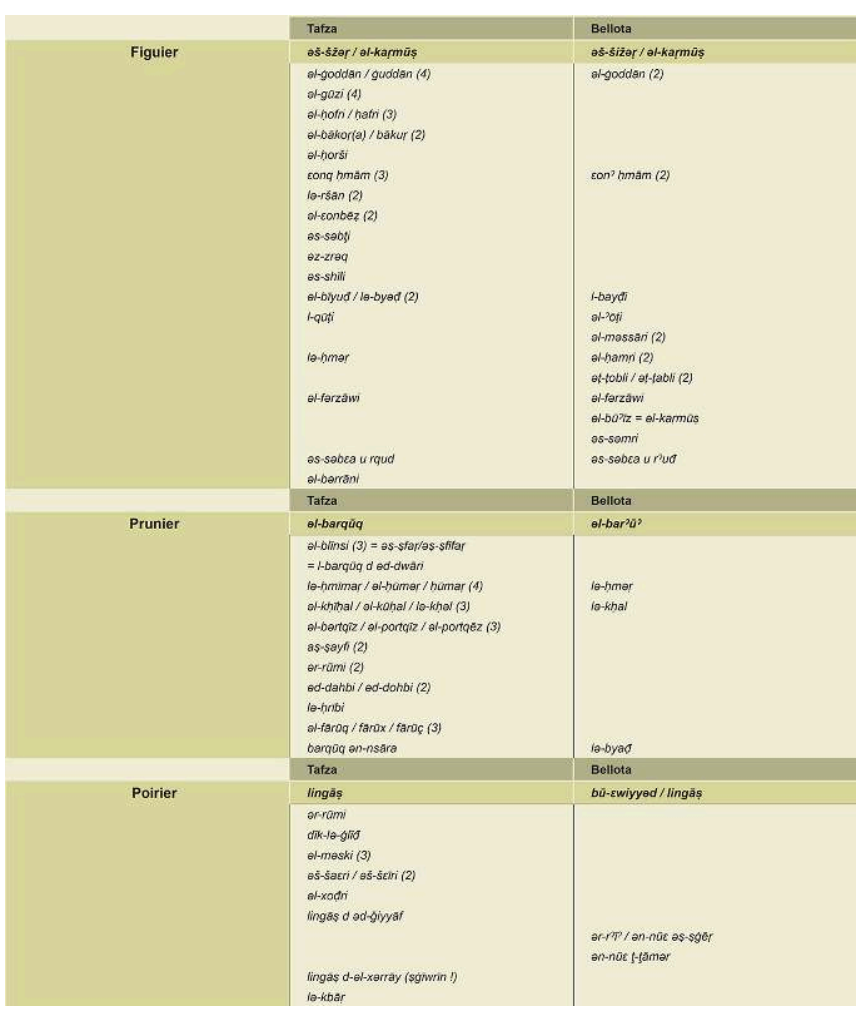
cohérents avec les données connues et confirment la toujours grande diversité dialectale de la région. Une diversité linguistique à laquelle fait écho l'agrobiodiversité du pays et la richesse de la taxinomie vernaculaire des variétés fruitières. Ce constat concorde avec les données déjà explicitées par les travaux sur le figuier de Hmimsa (2009) et Hmimsa et al. (2012 et 2017) et dont la richesse linguistique relève de champs sémantiques variés.

effet, toutes les variétés rencontrées portent un nom qui les différencie des autres. De la totalité des nominations recensées et analysées à l'échelle des trois fruitiers dans les deux sites, et à l'exception des termes qui n'ont pas de signification, nous avons constaté que la diversité variétale n'est pas pensée comme spécifiquement locale, et ce n'est pas l'objectif des agriculteurs. Si les arbres sont individuellement appropriés et que les agriculteurs les considèrent comme un bien, les variétés n'appartiennent à personne. Ainsi, en mettant de côté la nature des nomenclatures et en tenant compte seulement de leurs significations, nous avons constaté un regroupement des variétés selon :

- Des caractères morphologiques dont la désignation des variétés remonte à des générations passées. Aujourd'hui, les agriculteurs utilisent les mêmes désignations pour préserver les variétés reconnues et regrouper tout ce qui ressemble sur la base de caractères morphologiques. Ainsi, au niveau des deux sites, ces critères morphologiques sont des caractères liés directement à la couleur, l'aspect des feuilles ou du fruit, à la forme rappelant l'anatomie d'un animal, à la forme d'un autre fruit ou légume.

- Le partage des appellations reflétant leurs origines présumées en faisant référence à un groupe ethnique dont les agriculteurs supposent être à l'origine de la variété. Sinon

Revue d'ethnoécologie, Supplément 1 | 2017 
il reflète le lieu d'origine supposé de la variété et cela afin de la distinguer des autres variétés locales et lui attribuer un statut particulier.

- Le lien à un usage spécifique ou un usage dans une période spécifique.

51 Ainsi, les paysans, arrivent à ordonner et à classer l'ensemble de cette diversité, en imposant un mode de gestion qui est fondé non seulement sur la distinction entre les différentes variétés des différents arbres fruitiers (par externalisation à des cultures annuelles) mais également sur un processus adaptatif qui les amène à maintenir un grand nombre de variétés en utilisant leurs savoirs et connaissances des différents types de cultures. Ceci pour répondre à leurs besoins en autoconsommation d'une part (préférences gustatives, adaptation aux contraintes du milieu...), mais aussi pour surmonter les contraintes socioéconomiques à travers la commercialisation de la production, à l'exemple de L'Anjra dont les habitants sont surnommés Barqōqiyyen, leurs prunes étant commercialisées dans les différents centres urbains du pays Jbala.

Plus encore, du point de vue de la relation entretenue entre les habitants de la région jbala et leur environnement, on note l'importance centrale du figuier dans toute la région, Hmimsa et al. (2017), reflétée dans son nom générique local šžər, l'arbre par excellence. Mais on voit aussi qu'il n'est pas seul, le prunier a aussi une bonne place dans la zone septentrionale (Tafza) et, dans une moindre mesure, le poirier. Dans notre corpus, le nombre de termes ou de variétés citées apparaît nettement plus important, pour les trois fruitiers pris en compte, à Tafza. Ce déséquilibre est en partie dû aux profils des interviewé-e-s sur chaque site. À Tafza, les deux principaux interviewés ( $\mathrm{Si}$ A. et Si M.) sont deux hommes âgés qui ont une connaissance pratique, approfondie et ancienne de ces fruitiers. Tandis qu'à Bellota, la principale interviewée (F1), est une femme plus jeune et dont les connaissances et pratiques sont différentes, puisque l'olivier y a pris désormais plus de place (tant en termes de nombre d'arbres cultivés qu'en termes économiques).

Pour finir, la diversité des espèces fruitières est donc très importante. Toutefois, la diversité variétale est significativement plus importante pour les espèces comme le figuier, le prunier ou le poirier. Ces essences sont représentées par des variétés locales généralement très peu répandues et peu connues, et, souvent, rencontrées dans un site mais pas dans l'autre.

\section{BIBLIOGRAPHIE}

Aguadé J. 2008 - Árabe marroquí (Casablanca). In : Corriente F. \& Vicente Á. (Ed.), Manual de dialectología neoárabe. Zaragoza, Instituto de Estudios Islámicos y del Oriente Próximo : 289.

Aguadé J. 2003 - Estudio descriptivo y comparativo de los fonemas del árabe dialectal marroquí. Estudios de Dialectología Norteafricana y Andalusí (EDNA) 7 : 67-68.

Benitez Fernandez M. 2016 - Notes sur le sociolecte des jeunes d'Ouezzane (nord du Maroc). In : Grigore G. \& BiŢună G. Arabic Varieties: Far and Wide. Proceedings of the 11th International Conference of AIDA. Bucarest, Editura UniversitãŢii din București : 99-105. 
Caubet D. 2000-2001 - Questionnaire de dialectologie du Maghreb (d'après les travaux de W. Marçais, M. Cohen, G S. Colin, J. Cantineau, D. Cohen, Ph. Marçais, S. Lévi, etc.). Estudios de Dialectología Norteafricana y Andalusí (EDNA) 5 : 73-92.

Corriente F., Pereira Ch. \& Vicente Á (Ed.) 2017 - Encyclopédie linguistique d'Al-Andalus Vol. 2 : Dictionnaire du faisceau dialectal arabe andalou. Perspectives phraséologiques et étymologiques. Berlin/ Boston, De Gruyter : 1204 et 1301.

Dallet J.-M. 1982 - Dictionnaire kabyle-français. Paris, SELAF : 484.

Direction de la Statistique 2004 - Recensement Général de la Population et de l'Habitat (RGPH). Région de Tanger-Tétouan. http://www.hcp.ma/Recensement-general-de-la-population-et-de-lhabitat-2004_a633.html (Consulté le 06/05/2008).

Doré C. \& Varoquaux F. 2006 - Histoire et amélioration de cinquante plantes cultivées. Versailles, Quae, $812 \mathrm{p}$.

Guerrero Parrado J. 2014 - El dialecto árabe hablado en la ciudad marroquí de Larache. Zaragoza, Prensas de la Universidad de Zaragoza, $348 \mathrm{p}$.

Guy P. 1986 - Recherche et conservation des races et espèces en voie de disparition. In :

Fédération Française des Sociétés de Protection de la Nature, Agriculture et Environnement. Paris, Syros : 260-261.

Heath J. 2002 - Jewish and Muslim dialects of Moroccan Arabic. London/New-York, Routledge Curzon, $598 \mathrm{p}$.

Hmimsa Y. 2009 - L'agrodiversité de l'agroécosystème à l'arbre : cas du Rif (Nord du Maroc). Thèse de doctorat. Tétouan, Université Abdelmalek Essaadi - Faculté des Sciences.

Hmimsa Y. \& Ater M. 2008 - Agrodiversity in the traditional agrosystems of the Rif mountains (north of Morocco). Biodiversity: Journal of life on earth 9 (1-2) : 78-81.

Hmimsa Y., Aumeeruddy-Thomas Y. \& Ater M. 2017 - Lexique sur les figuiers : exemple des variétés nommées chez les Beni Ahmed (Jbala, Rif occidental). In : Vicente Á., Caubet D. \& NaciriAzzouz A. (Ed.), La région du nord-ouest marocain : Parlers et pratiques sociales et culturelles. Zaragoza, Prensas de la Universidad de Zaragoza : 265-274.

Hmimsa Y., Aumeruddy-Thomas Y. \& Ater M. 2012 - Vernacular Taxonomy, Classification and Varietal Diversity of fig (Ficus carica L.) Among Jbala cultivators in Northern Morocco. Human Ecology 40 : 301-313.

Iraqui Sinaceur Z. (Dir.) 1993 - Le dictionnaire Colin d'arabe dialectal marocain (arabe français). Rabat, Al Manahil, 2107 p. (8 volumes).

Marçais Ph. 1956 - Le parler arabe de Djidjelli (Nord constantinois, Algérie). Paris, Maisonneuve, 648 p.

Marçais W. 1911 - Textes arabe de Tanger. Transcription, traduction annotée, glossaire. Paris, Ernest Leroux : 229-230 et 459-460.

Martin G.J. 1995 - Ethnobotany: a Methods Manual. London, Chapman \& Hall, 268 p.

Messaoudi L. 1996 - Note sur l'affriquée /ğ/ dans le parler Jbala (nord du Maroc). Estudios de Dialectología Norteafricana y Andalusí (EDNA) $1:$ 167-175.

Messaoudi L. 1999 - Étude de la variation dans le parler des Jbala (nord-ouest du Maroc). Estudios de Dialectología Norteafricana y Andalusí (EDNA) 4 : 167-176.

Moscoso F. 2003 - El dialecto árabe de Chauen (N. de Marruecos) Estudio lingüístico y textos. Cádiz, Universidad de Cádiz, 382 p. 
Natividad E. 1998 - Le dialecte de Chefchaouen. In : Aguadé J., Cressier P. \& Vicente Á. (Ed.), Peuplement et arabisation au Maghreb occidental : dialectologie et histoire. Madrid/Zaragoza, Casa de Velázquez/Área de Estudios Árabes e Islámicos : 109-120.

Prémare A.-L. de 1993-1999 - Dictionnaire arabe-français, langue et cultures marocaines. Paris, L'Harmattan : 4874 p. (12 volumes).

Pruvost J. 2017 - Nos ancêtres les Arabes. Ce que notre langue leur doit. Paris, JC Lattès : 93.

Serhoual M. 2002 - Dictionnaire tarifit-français. Thèse de doctorat. Tétouan, Université Abdelmalek Essaadi - Faculté des Lettres et Sciences Humaines : 324.

Tilmatine M. 1999 - Substrat et convergences : le berbère et l'arabe nord-africain. Estudios de Dialectología Norteafricana y Andalusí (EDNA) 4 : 99-119.

Vicente Á. 2007 - Two cases of Moroccan Arabic in the diaspora. In : Miller C. et al. (Ed.) Arabic in the city. Issues in dialect contact and language variation. London/New York, Routledge :123-143.

Vicente Á. 2002 - Une interprétation sociolinguistique d'un dialecte de Jbala : les parlers féminin et masculin dans le dialecte d'Anjra. In : Youssi A. et al. (Ed.) Aspects of the Dialects of Arabic Today. Rabat, Amapatril : 336-344.

Vicente Á. 2000 - El dialecto árabe de Anjra (Norte de Marruecos) Estudio lingüístico y textos. Zaragoza, Universidad de Zaragoza : 28, 45, 47-49, 137.

Vicente Á. 1998 - Un dialecte de type montagnard au Maroc : le parler d'Anjra. In : Aguadé J., Cressier P. \& Vicente Á. (Ed.) Peuplement et arabisation au Maghreb Occidental. Dialectologie et histoire. Madrid/Zaragoza, Casa de Velázquez/Universidad de Zaragoza : 121-130.

Vicente Á., Caubet D. \& Naciri-Azzouz A. (Ed.) 2017 - La région du Nord-Ouest marocain : Parlers et pratiques sociales et culturelles. Zaragoza, Prensas de la Universidad de Zaragoza : $362 \mathrm{p}$.

Ziamari K. \& Barontini A. 2008 - Quelques éléments de description d'un parler jebli (Ourtzagh, Maroc). Estudios de Dialectología Norteafricana y Andalusí (EDNA) 12 : 43-59.

\section{ANNEXES}

Extraits du corpus

Tafza :

«Y : u b nəsba l:: əš-šžər ašnu l-ənwāe lli عənd-kum?

et par rapport au figuier quelles sont les variétés que vous avez?

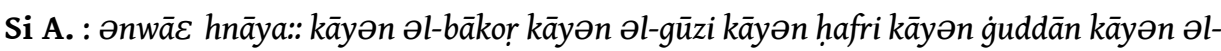
horši kāyən عonq ḥmām.. kāyən lə-ršān kāyən e:: Eonbēz e:: kāyən e:: bəzzā:::f kāyən əs-səbŢi k-yqūlu l-u.. kāyən bəzzāf əl-unwāe hnāya. əl-ḥaqq dāba dìk-l-ənwāe hādu lli bqāw hnāya. l-haqqa bəzzāf hūwa l-hafri u l-gūzi u l-g்uddān u l-bākor des variétés ici il y a la figue fleur, il y a əl-gūzi il y a hafri, il y a ǵuddān il y a ol-ḥorši, il y a coup de pigeon, il y a lə-ršān il y a euh عonbēz euh il y a euh beaucoup de variétés ici. Il y a le ceuti comme on l'appelle. Il y a beaucoup de variétés ici. En vérité maintenant ce sont ces variétés qui restent ici. Vraiment il y en a beaucoup c'est l-hafri et l-gūzi et $l$ guddān et la figue fleur (...) 
$\mathbf{Y}:$ u l-bərqū $q l^{2}$ anwāe dyāll-barqūq

et les pruniers les variétés des prunes

Si A. : a l-barqūq bəzzā̄:::f kāyən portqīz kāyən e:: ər-rūmi kāyən əl-hūmər kāyən əd-dahbi kāyən e:: lə-ḥribi kāyən bəzzāi:::fd əl- ${ }^{2} a n w a \bar{\varepsilon}$ hna

ah les pruniers il y en a beaucoup il y a portqiz il y a l'européen il y a le rouge il y a le doré il y a lə-ḥrỉbi il y a beaucoup de variétés ici.

$\mathbf{Y}:$ ol-blinsi

Si A. : blīnsi. blīnsi đa hāđa hūwa lli::

blinsi oui c'est celui-ci qui...

(...)

Y : dāba l-blīnsi hūwa lli ka-ysəmmīw-əh fblāșa uxra š-šEīri

maintenant l-blīnsi c'est celui qu'on appelle ailleurs š-šcīri.

Si A. : la. hāđāāk bwəh̆d-u hāđāke. kāyən wāh ā āxor ka-yqūlu š-šEīiri. la l-blinnsi k-yzi byəț. kayțēb hūwa l-uwwlāni

non. Celui-là c'est un autre. il y en a un autre qu'on dit š-šEiri. non l-blinsi devient blanc. C'est lui qui mûrit en premier.

A : əš-šEīiri k-ykūn zrəq šwìya u șgièr

əร̌-šEiri il est un peu bleu et petit.

Si A. : eh. blīnsi ka-yțīb hūwa l-uwwlāni hā hūwa awāxēr šhar əs-səŢ̧̧a ka-ybda yțīb. ka-ybda yțīb u șəbhān lḷāh əl-bərqūq dyāl əl-blinsi:: kūl lli bg̀iti kūl dìk-əl-bārāka dyāl-um u l-mākla

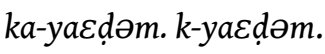

oui. blinsi c'est lui qui mûrit en premier vers la fin du mois de juin il commence à mûrir. Il commence à mûrir et Dieu soit loué la prune du blinsi.. Mange ce que tu veux, mange leur bénédiction et la nourriture, elle (la prune) devient énorme. Elle devient énorme.

Y : u hūwa lli ka-ydir ən-nəsma?

c'est lui qui exhale un parfum?

Si A. : hūwa lli ka-ydìr ən-nəsma hūwa u wāḥd-ən-nōe āxor hādāk əl-fārūq

c'est lui qui exhale un parfum lui et une autre variété celui-là c'est əl-fārūq (...)

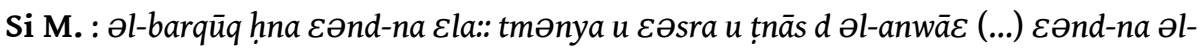
uwwliya əl-blinsi

le prunier nous nous en avons autour de huit et dix et onze variétés (...) nous avons le premier al-blinsi

Si A. : hūwa lli ka-yțēbl-uwwlāni

c'est lui qui mûrit en premier.

Si M. : hūwa lli ka-yțēb l-uwwli. ¥ānīyān əl-fārūç. ¥ālì̄ān

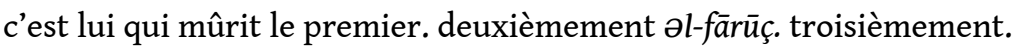

Si A. : $ə r-r \bar{m} m i$

l'européen.

Si M. : la bāqi. əl-kūḥal. rāäbīeān əl-ḥūmar. xāmīsān ər-rūmi. sādīsān lə-ḥribi. sābīeān əlportqiz 
non il en reste. le noir. quatrièmement le rouge. cinquièmement l'européen. sixièmement lo-hrïbi. septièmement əl-portqiz.

Si A. : əd-dahbi

le doré.

Si M. : fāmin̄ān əd-dohbi. zīd ā Sidi A.

huitèmement le doré. continue Monsieur A.

Si A. : e:: qūll-i xxx hād-əl-barqūq əṣ-șfar. ās ka-yqūlu l-u hādāk ?

euh dis-moi xxx cette prune jaune. comment on l'appelle celle-là ?

Si M. : e::h barqūq hna nqūlu l-u l-barqūq d əd-dwāri

oui une prune ici on l'appelle la prune des écus.

(...)

Si A. : u blinsi lə-hmar

et le blinsi rouge.

Si M. : blinsi lə-hmar. iyyeh

le blinsi rouge. oui

(...)

Si M. : $\varepsilon ə n d-n a ~ l-b a ̄ k u r$

nous avons la figue fleur (l-bākur).

A : əl-bākur Eāwtāni šnu l-anwāe d əl-bākur?

la figue fleur aussi quelles sont les variétés de figue fleur?

Si M. : hā hūma fin hūma hā hìya. hād-əl-bākur ka-yțîbu hūma l-uwwlīn

les voilà c'est elle c'est tout. ces figues fleurs c'est elles qui mûrissent les premières.

A : yqūlu l-u l-bayḍa

on l'appelle la blanche.

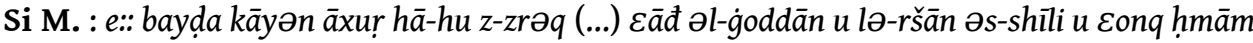
euh blanche il y en a une autre la bleue (...) et après əl-goddān et lə-ršān, əs-shili et le coup de pigeon.

Si A. : əl-ḥafri

əl-hafri.

Si M. : u l-hafri u l-bìyự u l-qūṭi

et $l$-hafri et le blanc et $l-q u \bar{u} t i$.

Si A. : u l-gūzi

et $l$-gūzi.

Si M. : u l-gūzi

et $l$-gūzi.

Si A. : u l-Eonbēz

et $l$-Eonbēz.

Si M. : u l-Eonbēz iyyeh iyyeh

et $l-\varepsilon$-Enbezz oui oui.

(...) 


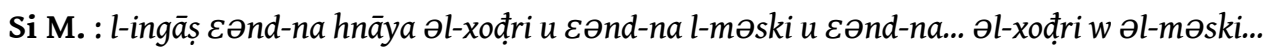
șāfi hādāhəm lli عənd-na șāfi عənd-na žūž d l-anwāe

le poirier nous avons ici le vert et nous avons le muscat et nous avons... le vert et le muscat... c'est tout c'est ceux-là que nous avons c'est tout nous avons deux variétés.

$\mathbf{Y}:$ əૅ̌̌š̌̌ìi?

le šcīi?

Si M. : u əš-šEīri ḷ̂āh yrḥam bābā-k iyyeh. șāfi

et le šcīri Dieu bénisse ton père oui. c'est tout.

$\mathbf{Y}: u$ ǧiyyāf

et ğiyyāf.

Si M. : eh. l-ingāṣ d əd-ğiy\# (...) l-ingāṣ d əd-ğiyyāf. iyyeh oui. la poire du ğiy\# (...) la poire du ğiyyāf. oui.

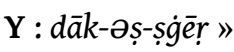

la petite-là.

Bellota :

F1 : la, āktārīya n-nās dāba lli yg்arsu əš-šižər dyāl-əm. mm non, c'est la plupart des gens maintenant qui cultivent leurs propres figuiers. hum. (...)

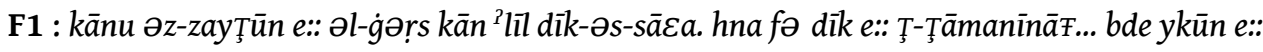

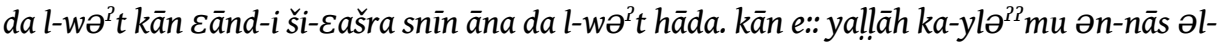

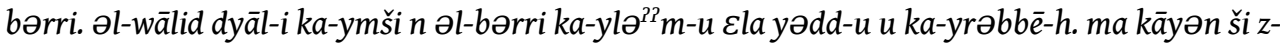
zayŢūn ma kāyən š əl-būŢor d əz-zayŢūn bhāl e:: hāđ e:: s-sīnin hāđi

il y avait des oliviers euh il y avait peu de plantations à ce moment-là. ici dans les.. années 80 .. ça a commencé à.. en ce temps moi j'avais environ dix ans. c'était euh.. les gens greffaient à peinel'olivier sauvage. mon père il allait greffer l'olivier sauvage à la main et il le faisait pousser. il n'y avait pas d'olivier pas de boutures d'olivier comme ces années-ci.

(...)

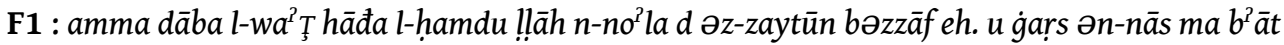
ši ši-blāṣā Eənd-na hna f əl-mənțā̄ a bla ġoṛs. āktārīya mə ġợs əz-zaytūn. āktārīya. mais maintenant de nos jours Dieu merci il y a beaucoup de plants d'oliviers oui. et les plantations des gens il n'y a pas un seul endroit chez nous ici dans la région sans plantation. La plupart ce sont des oliviers. la plupart.

(...)

F1 : eh dāba əz-zayŢūn kŢəṛ. eh əz-zayȚūn kŢər. b nəsba l-manță $\bar{a}^{2}$ dyāl-na əz-zayȚūn hūwa lli... hūwa lli dāba əl-mədxōl d hā-l-fillāh d əl-manța $\bar{a}^{2}$ a dyāl-na. hūwa l-mədxōl d əs-sāna kull-

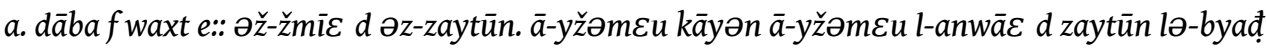
e:: lə-khal (...) wāhəd-əl-mədxōl dyāl əs-sāna kull-a. b nəsba l-manțā a dyāl-na hnāye. (...) əd-

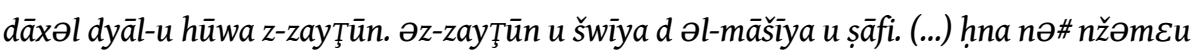

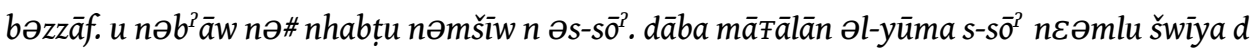

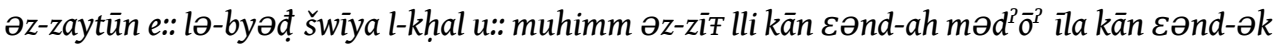

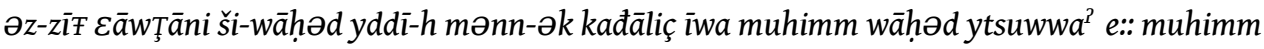
mən e:: l-manţūš dyāl-u. mə l-manŢūš dyāl-u » 
oui maintenant il y a plus d'oliviers. oui plus d'oliviers. par rapport à notre région c'est l'olivier qui... c'est lui qui constitue le revenu de l'agriculteur de notre région. c'est le revenu de l'année entière. maintenant c'est le moment de la récolte des olives. ils récoltent il y en a qui récoltent l'olive verte (claire) euh l'olive noire. c'est un revenu pour toute l'année. en ce qui concerne notre région ici. son revenu est constitué par les olives. les olives et un peu le bétail et c'est tout (...) nous nous en récoltons beaucoup. et nous nous sommes mis à descendre au marché. maintenant par exemple aujourd'hui au marché on fait un peu d'olives vertes (claires) un peu d'olives noires et. bref l'huile qui a été pressée si tu as de l'huile aussi quelqu'un te l'emmène aussi donc quelqu'un va vendre au marché euh en fonction de l'extraction qu'il en a tiré. à partir de son extraction. »

\section{NOTES}

1. jbāla est le pluriel de jəbli, montagnard, ce terme désigne à la fois la région au nord-ouest de la chaîne du Rif, ses habitants, et les parlers arabes locaux (cf. Vicente et al. 2017).

2. «Ceux des prunes », mot arabe formé avec un suffixe de pluriel berbère (cf. Tilmatine 1999).

3. Au nord du Maroc, c'est une chaîne montagneuse alpine en forme d'arc concave ouvert vers la Méditerranée, correspondant au prolongement sud de la cordillère Bétique (Andalousie, Espagne), et qui est la principale chaîne montagneuse de la partie septentrionale du pays.

4. Nous n'avons pas analysé le cas de la vigne malgré la diversité nommée, car en réalité, la présence de la vigne est très minime. À cause des vagues de phylloxera qui ont eu lieu, la population n'a pas replanté la vigne et il ne reste que des pieds éparpillés dans le paysage sur chaque site.

5. «(...) hay dialectos que hoy en día presentan interdentales que diacrónicamente no tienen nada que ver con las del árabe clásico y que se deben a una fricatización secundaria de oclusivas por influencia del bereber: suele darse en posición final o intervocálica y es un fenómeno característico de los dialectos de Jebala y de algunas ciudades del norte de Marruecos como por ejemplo Chauen. ».

6. Voir la remarque sur les interdentales dans $/ \mp /$, ci-dessus.

7. « la asimilación del artículo definido /l-/ ante /ž/ hace que la aparición de esta geminación y su consiguiente disimilación sean muy frecuentes, ejemplos: $d$-ğlăləb « las chilabas » (...) ».

8. Littéralement "l'arbre", le figuier étant l'arbre fruitier le plus important en termes de production et de consommation, c'est l'arbre par excellence (Hmimsa 2009, Hmimsa et al. 2012, Hmimsa et al. 2017).

9. On le retrouve aussi bien en arabe andalou (Corriente et al. 2017), ou en arabe moderne standard qu'en berbère rifain (« ғamešmašғ; rmešmeš », Serhoual 2002) ou kabyle («lmecmac / amemmac », Dallet 1982).

10. nǐš est ainsi mentionné dans Colin (Iraqui Sinaceur 1993) et Premare (1993) comme particulier à la région. On le retrouve en andalou, et il viendrait du " Néo-persan nišu/e " sorte de prune " " (Corriente et al. 2017). Ainsi, le cheminement du sémantisme de niš (de prune à abricot) a été l'inverse de celui de bərqūq (d'abricot à prune).

11. Littéralement. 


\section{RÉSUMÉS}

Le pays occupe la majeure partie de la chaîne du Rif occidental et central, depuis le détroit, jusqu'au pays de l'Ouergha au sud, et ne comprend pas les plaines et plateaux atlantiques. Il s'agit d'un espace montagnard occupé par une population rurale relativement dense et dont l'activité principale est l'agriculture. C'est une agriculture traditionnelle marquée par la pratique d'une polyculture de subsistance dans le cadre d'un système de production agro-sylvo-pastoral et qui comprend des unités paysagères particulières. Les particularités physiques, naturelles, socioéconomiques et historiques du pays Jbala ont créé des conditions qui ont contribué à maintenir un certain nombre de cultures et de pratiques rares.

Dans le cadre du Projet International de Coopération Scientifique (PICS) franco-marocain - La Montagne et ses Savoirs - nous avons recueilli (en octobre 2013 et en avril 2014) un corpus d'interviews auprès d'agriculteurs, de marchands et plus largement d'habitants de la région (principalement à Anjra et Bellota).

Cet article vise à présenter les résultats d'une comparaison entre la diversité des dénominations et la diversité linguistique entre le nord (Anjra) et le sud (Bellota) du pays Jbala, à travers une part de la taxinomie vernaculaire concernant les fruits et les arbres fruitiers.

The Jbala region is located mostly on the western and central Rif's mountain chain, from the Straits of Gibraltar to, south, the Ouergha (without the atlantic plains and plateau). It is a mountain area, inhabited by a rural and quite dense population, whose principal economic activity is agriculture. A traditional agriculture mostly characterised by the use of a subsistence mixed farming as part of a agro-sylvo-pastoral system of production which constitutes specific landscapes. Physical, natural, socio-economic and historic particularities created favorable conditions maintaining several rare cultivated plants and practices.

Thanks to the international scientific cooperation project (PICS) «La Montagne et ses Savoirs ", we have collected (between October 2013 and April 2014) a series of interviews with farmers, merchants or inhabitants of the region, in two sites: Tafza and Bellota.

We present here the results of a modest comparison between the agro-biological diversity and the linguistic diversity, through some of the vernacular taxinomy concerning fruits and fruit trees.

\section{INDEX}

Keywords : Jbala region, agrobiodiversity, linguistic diversity, nomenclature, Arabic mountaineers speaking, fruit trees

Mots-clés : pays Jbala, diversité linguistique, agrobiodiversité, nomenclature, parlers arabes montagnards, arbres fruitiers

\section{AUTEURS}

\section{ALEXANDRINE BARONTINI}

Maître de conférences à l'Inalco (Paris), arabe marocain (sociolinguistique et anthropologie), EA 4092 LaCNAD. INALCO, 65 Rue des Grands Moulins, 75013 PARIS

alexandrine.barontini@inalco.fr 


\section{YOUNES HMIMSA}

Enseignant chercheur à l'Université Abdelmalek Essaâdi, Département des Sciences de la Vie (Biologiste et ethnobotaniste, Membre du laboratoire de Botanique appliquée (Equipe BioAgrodiversité)), Faculté

Polydisciplinaire de Larache. B.P. 745, Poste Principale, Larache 92004 MAROC

hmimsayounes@gmail.com 